\title{
Analysis of the Europeans' Attitudes towards Vaccination in the Context of the Covid-19 Pandemic - Implications for Public Health Policy ${ }^{1}$
}

\author{
Gina Cristina DIMIAN* - Maria Denisa VASILESCU** - Simona Andreea \\ APOSTU ${ }^{* * *}$ - Mirela Ionela ACELEANU**** -Josef JABLONSKY*****
}

\begin{abstract}
In the current context of the Covid-19 pandemic, health systems worldwide have been subjected to hitherto unknown challenges. Public health policy makers are urged to find the best solutions to mitigate the effects of the pandemic. Vaccination is seen, more than ever, as the main medical solution to save lives, although in recent times many countries have seen an increase in their citizens' hesitation to get vaccinated. The aim of our research is to analyze Europeans' attitudes towards vaccination and the factors that influence this attitude, both in terms of individual profile and differences between groups of people and between countries. The results confirmed that a positive attitude towards vaccination increases an individual's chances of getting vaccinated and that the vaccination depends on the socio-demographic characteristics of the individual.
\end{abstract}

Keywords: attitudes towards vaccination, Covid-19 pandemic, the European countries, statistical and econometric methods

JEL Classification: C10, I15, O33

DOI: https://doi.org/10.31577/ekoncas.2021.10.01

\footnotetext{
* Gina Cristina DIMIAN, corresponding author, Bucharest University of Economic Studies, Department of Statistics and Econometrics, Bucharest, Romania; e-mail: gina.dimian@csie.ase.ro

** Maria Denisa VASILESCU, Bucharest University of Economic Studies, Department of Statistics and Econometrics, Bucharest, Romania; National Scientific Research Institute for Labour and Social Protection, Bucharest, Romania; e-mail: maria.vasilescu@csie.ase.ro

*** Simona Andreea APOSTU, Bucharest University of Economic Studies, Department of Statistics and Econometrics, Bucharest, Romania; Institute of National Economy - Romanian Academy, Bucharest, Romania; e-mail: simona.apostu@csie.ase.ro

**** Mirela Ionela ACELEANU, Department of Economics and Economic Policy, Bucharest University of Economic Studies, Bucharest, Romania; e-mail: aceleanu_mirela@yahoo.com

$* * * * *$ Josef JABLONSKY, University of Economics Prague, Department of Econometrics, Prague, Czech Republic; e-mail: Jablon@ vse.cz

${ }^{1}$ This work was partially supported by a grant of the Romanian National Authority for Scientific Research and Innovation, CNDS-UEFISCDI, PNCDI III Program - Project Understanding and Modelling Time-space Patterns of Psychology-related Inequalities and Polarization, PN-IIIP4-ID-PCCF-2016-0084.
} 


\section{Introduction}

The outbreak of the Covid-19 pandemic uncovered some of the main challenges that public health policy needs to address (Weeramanthri and Bailie, 2015): the health of the planet and of the environment, a global health system, wellbeing and healthy life/aging and valuable investments in public health. The pandemic deepened ethnic, economic, social, and gender inequalities given that vulnerable groups and countries have been the most impacted (Lamber et al., 2020). The collateral effects of the recent pandemic are equally severe: aggravation of the food crisis and the possible onset of an education crisis in low-income countries, closure of many businesses, and job loss in all countries of the world, regardless of the level of development (Lamber et al., 2020).

The consequences of the ongoing pandemic cannot be yet accurately measured, but it is anticipated to be very severe and with multiple ramifications. In this context, researchers, politicians, and governments are urged to find targeted solutions to mitigate its effects. The main solution to the health crisis is vaccination, but how effective it will be remains to be demonstrated.

Nowadays, World Health Organization estimates reveal that vaccines save between 1 and 3 million lives each year (WHO, 2018), and will save another 25 million people in the next decade (Rappuoli, 2014), fact that is supported by high vaccination rates and equal access to vaccination for all ages and populations. Vaccination has made beneficial contributions, both medically and socio-economically, studies indicating that improving health leads to long-term economic growth, strengthening macroeconomic stability, and improving educational outcomes (Bloom, Canning and Jamison, 2004; Belli, Bustreo and Preker, 2003).

Of paramount importance is the vaccination strategy, with adequate vaccine allocation criteria such as: utility (immunization of people at high risk of morbidity and mortality), risk of acquiring infection and of negative societal impact (immunization of front-line healthcare and of other essential workers), equal regard, mitigation of health inequities, fairness and transparency (National Academies of Sciences, 2020).

Critical for the success of vaccination is also the attitude of the population, given that in recent years, several studies have shown an increase in vaccine hesitancy. Vaccine hesitancy, defined as 'the delay in acceptance or refusal of vaccines despite the availability of vaccination services, is a complex phenomenon that tends to be 'context-specific, varying across time and place and with different vaccines' (Dubé et al., 2014). The analysis of the trends in vaccine confidence in the countries of the world has shown the existence of differences between them, with many countries in which the confidence in vaccination has decreased the motivation of such an evolution remaining to be investigated 
(de Figueiredo et al., 2020). People's attitudes towards vaccination can vary even within a country, at the regional level or even more localized (Dubé et al., 2014).

Among the factors that determine the attitude towards vaccination, the most important proved to be the beliefs related to the importance, safety, and effectiveness of vaccines (de Figueiredo et al., 2020). It seems that rather trust or lack of trust in the institutions involved in vaccination influences the population's behavior related to vaccination, less influence having factors such as lack of awareness or misinformation (Yaqub et al., 2014). The Internet and the media are two other sources of information that have been shown to contribute to shaping the population's attitude toward vaccination (Yaqub et al., 2014).

The persistence of misconceptions about vaccination (like the parents' view that vaccines cause autism spectrum disorder in children), although scientific evidence proves otherwise, requires an internationally coordinated strategy for rapid, easy-to-understand and reliable communication of scientific evidence concerning vaccination (Salmon and Dudley, 2020). In this sense, smart technology can offer many opportunities both in terms of increasing vaccine safety and confidence.

Thus, disentangling the factors that influence individuals' perceptions concerning vaccination and understanding the grounds of heterogeneity among social categories and countries are of utmost importance for a successful exit from this global health crisis, given that virus transmission has no borders. Although the European Union should act as a unitary entity, local specificity cannot not be neglected (Lacko et al. 2020). The aim of our research is to analyse Europeans 'attitudes towards vaccination and the factors that influence this attitude. There are considered two research hypotheses about the vaccination: 1 . Self-vaccination is driven by individuals' perceptions about vaccination and their socio-demographic characteristics. 2. There are larger differences between groups of people and less between countries in terms of knowledge, attitudes, and behaviour towards vaccination.

The rest of the paper is organized as follows: the next section reviews the findings of the recent literature in terms of vaccination as a solution to Covid-19 crisis, the third part of the paper presents the variables, data and methods applied to verify the research hypotheses, the fourth section describes the results of the empirical analysis, while the final discussions and conclusions are the subject of the last parts of the paper.

\section{Literature Review}

The pandemic caused by Covid-19 has affected and continues to affect the world population. Covid-19 has hit health systems, has disrupted people's lives and has slowed down all economic activities. 
Containing COVID-19 pandemic and reducing its effects is a considerable challenge for all countries. Yet, they have been exposed to the virus at different times and have a different number of cases and fatalities, given that each country has responded differently to the COVID-19 outbreak, at varying speeds and with different measures (Primc and Slabe-Erker, 2020).

The adoption of containment measures depends not only on a health risk assessment but also on an estimate of possible economic loss. The biggest challenge in the decision-making in preventing health risks is to find a balance between reducing the risk of viral transmission and the economic costs (Anderson et al., 2020).

Among the decision makers, we find both a minimalist approach to the Covid-19 crisis in terms of impact on the economy, but also a maximalist approach that places the coronavirus crisis at the key of state interventionism, with effects on individual freedom. The efficiency of economic measures is changing, the measures that worked in the past may be without effect in the present, because they depend on factors such as economic confidence and the success of medical measures (Marinescu, 2020).

The COVID-19 pandemic revealed that the healthcare systems' preparedness for sudden shocks and health problems in elderly people needs more attention from the public and politicians, regardless of income level (Giang, Vo and Vuong, 2020). In any country, timely and strong cooperation between the government, civil society, and the private sector is important in strengthening confidence in the fight against the public health crisis (La et al., 2020).

In this context, innovation plays a significant role in recovery through international collaboration to find a vaccine and to identify the most appropriate therapies. Vaccination has been practiced systematically since 1796, when Edward Jenner developed the first smallpox vaccine. Vaccinations and antibiotics are considered the most important medical contributors to the growth of the world's population after 1945 (Gherghina et al., 2019).

However, even before Covid-19, specialized studies showed that influenza vaccination was carried out too rarely and thus vaccine coverage rate, both in the general population and in risk groups, was at an unsatisfactorily level (NitschOsuch, Jagielska and Brydak, 2020).

Among the reasons behind the acceptance of vaccination within the general population are healthcare professionals' advice; advice from friends, family, or colleagues; self-protection, others protection, and awareness of illness (Yaqub et al., 2014). The most commonly cited reason for general population hesitancy towards vaccination is safety concerns. Other reasons for hesitation are lack of awareness, low perceived severity of illness and belief in alternative medicine, lack of knowledge, and distrust in pharmaceutical companies (Yaqub et al., 2014). 
An analysis of the knowledge, attitude, and behaviour of Italian healthcare workers towards the vaccines recommended by the Ministry of Health showed that they have a positive attitude towards the usual vaccinations, for example, for Hepatitis B virus (HBV) and tuberculosis (TB), but for other vaccinations the interest is low (La Torre et al., 2017). Moreover, the attitude towards vaccination also depends on the stress generated by a new virus, which spreads quickly, with serious effects. In this case, the side effects of vaccination may be less than the benefits (La Torre et al., 2017).

Given that in the event of a pandemic, such as Covid-19, vaccination plays an important role, understanding the individual's attitude towards vaccination is of tremendous importance. The attitude of the population towards vaccination depends on several characteristics such demographic and socio-economic status, but confidence in vaccines and trust in healthcare workers seem to be the most important determinants of vaccine uptake (Yaqub et al., 2014). In this regard, efforts should be made to improve the knowledge of official vaccination recommendations in the general population and to reduce common misconceptions about vaccinations (Akmatov et al., 2018).

\section{Data and Methodology}

We used in our research the data retrieved from the Eurobarometer 91.2 (European Commission, 2019) about 27 countries in the European Union and Great Britain (approximately 27,500 observations). The logistic regression model and TwoStep Cluster algorithm have been applied to test the research hypotheses:

H1: Self-vaccination is driven by individuals' perceptions about vaccination and their socio-demographic characteristics.

H2: There are larger differences between groups of people and less between countries in terms of knowledge, attitudes, and behaviour towards vaccination.

To test the first hypothesis, according to which vaccination depends on the individual's perceptions about vaccination as well as on the socio-demographic characteristics, we estimated a logistic regression model, the dependent variable being self-vaccination in the last five years ( $1=$ yes, $0=$ not mentioned).

The independent variables were chosen in accordance with the purpose of the research and can be grouped into two categories: i) Variables describing the individual's attitude regarding vaccination: vaccine effectiveness; vaccine knowledge; the importance of routine vaccination, the opinion that vaccination is important only for children, is important for self and others or it matters for those that cannot be vaccinated, the opinion that not being vaccinated can cause serious health issues; sources of information on vaccination (family, friends, doctor, 
healthcare workers, pharmacists, health authorities, online social networks, other internet sites); the opinion that vaccination program is better coordinated at international, European, national or local level or must be exclusively a personal choice; media sources of information about vaccination (TV, radio, newspapers, online social networks or other internet sites); actions that should be done to improve access to healthcare (more research, more affordable treatments, more medical staff in rural areas, more doctors, more money allocated to healthcare, more medical establishments); and ii) Socio-economic variables: age, gender, level of education, marital status, employment status, type of community, difficulties in paying bills (proxy for income).

Regression is a commonly used statistical method in data processing and consists in relating a dependent variable $y$ with one or more explanatory variables $x_{1}, x_{2}, \ldots, x_{p}$, called predictors. The binary logistic regression corresponds to the case where the dependent variable contains only two classes, the individuals being described by the presence or absence of a given characteristic. As the dependent variable can only take two values, coded, for example, $y=1$ (success) and $y=0$ (failure), the objective is to model, as a function of $x$, the probability of belonging to one of the two categories. We denote this probability $\pi\left(x_{i}\right)$, or simply $\pi$ (Gillet, Brostaux and Palm, 2010).

The probabilities $\pi\left(x_{i}\right)$, however, evolve in a nonlinear manner as a function of $x_{i}$. Therefore, the use of a linear model expressing $\pi$ as a function of $x$ and estimated by ordinary least squares is not an adequate solution. For this reason, we perform a transformation of the probability of success $g(\pi(x i))$. This transformation is called a link function, and thereafter will be denoted simply by $g$.

Several link functions exist but the most commonly used is the logit function, because it leads to a simple interpretation of the results, but also for theoretical reasons (Collett, 2002):

$$
g=\log i t(\pi)=\ln \left(\frac{\pi}{1-\pi}\right)
$$

The regression model can be written:

$$
g=\alpha+\beta \cdot x+\varepsilon
$$

where $\alpha$ and $\beta$ are parameters to be estimated, most often by the maximum likelihood method. The inverse transformation then makes it possible to find the estimated probabilities as a function of $x$ :

$$
\pi=\frac{\exp (g)}{1+\exp (g)}
$$

which are always between 0 and 1 . 
For a given value $x_{i}$, the ratio between the probability of success $\pi$ and the probability of failure $1-\pi$ is most often referred to as odds. It is equal to:

$$
\frac{\pi}{1-\pi}=\exp (g)
$$

If we consider the ratio between the odds relating to $x_{i}+1$ and to $x_{i}$, we define the odds ratio, which is directly related to the regression coefficient $\beta$ :

$$
\frac{\pi\left(x_{i}+1\right) /\left[1-\pi\left(x_{i}+1\right)\right]}{\pi\left(x_{i}\right) /\left[1-\pi\left(x_{i}\right)\right]}=\exp (\beta)
$$

The odds ratio is never negative, but has no upper bound. A value equal to unity means that the odds for $x_{i}$ are equal to the odds for $x_{i}+1$. In this situation, the explanatory variable therefore has no effect on the odds. An odds ratio less than unity corresponds to a negative regression coefficient and means that the probability of success decreases as $x$ increases. An odds ratio greater than unity corresponds to a positive regression coefficient and means that the probability of success increases as $x$ increases.

In the second stage of our research, we investigated if there are differences between groups of citizens and between European countries in terms of the knowledge, attitudes, and behaviour related to vaccination. We asked ourselves if attitudes can be group-dependent or country-dependent, and in order to test this hypothesis we first grouped the citizens and then the countries using TwoStep Cluster Analysis. We have evaluated the robustness of our results with the help of the Silhouette measure (which can be used in assessing the validity of clustering, Rousseeuw, 1987).

3 selected items have been included in the TwoStep Cluster Analysis: vaccine _knowledge (high, medium, or low), vaccine_effectiveness (in preventing infectious diseases like flu, measles, polio, hepatitis, meningitis and tetanus: definitely, probably, probably not, not at all) and self_vaccination (yes/no).

First, we have run TwoStep Cluster algorithm with all 27,500 observations and created groups of people with similar characteristics. Secondly, for each of the 28 countries and based on the previously mentioned 3 items, we computed a score in which the answer categories were weighted with the percentage of respondents corresponding to each category. In this case, the TwoStep Cluster Analysis (TSCA) was applied for grouping the 28 European countries.

We selected TwoStep Cluster methodology due to its advantages: i) it can be applied on very large data sets (our data set comprises 27,500 observations); ii) it is can be used both for continuous and categorical variables (we employed 3 categorical variables); iii) it groups the observation in a two-step process and 
automatically select the appropriate number of clusters (the number of clusters can demonstrate the heterogeneity of our sample).

The TSCA is based on a pre-cluster and cluster procedure. The precluster step is a sequential procedure consisting in the observation of each case in the order that it has been recorded. In function of a distance criterion, the observed case is either allocated to a previously formed cluster or forms a new one. This procedure consists in creating a modified cluster feature tree (CFT) with levels of nods and a number of entries for each node. The entries are defined by certain features: number of observations, mean and variance (for continuous variables), and counts for the categories of categorical variables (IBM, 2020). The cluster step uses the resulted subclusters from the previous step and creates the final clusters by means of a clustering method such as agglomerative hierarchical algorithm.

The number of clusters is determined automatically in a two-step procedure which starts with the identification of an initial estimate based on the computation of the BIC (Schwarz's Bayesian Criterion) or AIC (Akaike Information Criterion) measures. The final number of clusters is given by the largest increase in distance between the two closest clusters (IBM, 2020).

TSCA can be performed by computing two types of distances: log-likelihood distance and Euclidian distance.

The log-likelihood distance can be used both for continuous and categorical variables and is defined as (Bacher, Wenzig and Vogler, 2004):

$$
d(i, s)=\xi_{i}+\xi_{s}-\xi_{(i, s)}
$$

where $d(i, s)$ is the distance between cluster $i$ and cluster $s$.

The dispersion within a certain cluster contains two types of variances: the variance of continuous variables and variance of categorical variables or the entropy:

$$
\begin{gathered}
\xi_{i}=-n_{i}\left(\sum_{j=1}^{p} \frac{1}{2} \log \left(\hat{\sigma}_{i j}^{2}+\hat{\sigma}_{j}^{2}\right)-\sum_{j=1}^{q} \sum_{l=1}^{m_{j}} \hat{\pi}_{i j l} \log \left(\hat{\pi}_{i j l}\right)\right) \\
\xi_{s}=-n_{s}\left(\sum_{j=1}^{p} \frac{1}{2} \log \left(\hat{\sigma}_{s j}^{2}+\hat{\sigma}_{j}^{2}\right)-\sum_{j=1}^{q} \sum_{l=1}^{m_{j}} \hat{\pi}_{s j l} \log \left(\hat{\pi}_{s j l}\right)\right) \\
\xi_{(i, s)}=-n_{(i, s)}\left(\sum_{j=1}^{p} \frac{1}{2} \log \left(\hat{\sigma}_{(i, s) j}^{2}+\hat{\sigma}_{j}^{2}\right)-\sum_{j=1}^{q} \sum_{l=1}^{m_{j}} \hat{\pi}_{(i, s) j l} \log \left(\hat{\pi}_{(i, s) j l}\right)\right)
\end{gathered}
$$

where for any cluster $v(v=i, s,\langle i, s\rangle)$. 


$$
\hat{\pi}_{v j l}=\frac{N_{v j l}}{N_{v}}
$$

and $n_{v}$ represents the number of records in cluster $v, p$ is the number of continous variables $x_{j}(j=1,2, \ldots, p), q$ is the number of categorical variables $a_{j}(j=1,2$, $\ldots, \mathrm{q}), m_{j}$ represents the number of categories of variable $a_{j}\left(\mathrm{l}=1,2, \ldots, m_{j}\right) . \hat{\sigma}_{j}^{2}$ and $\hat{\sigma}_{v j}^{2}$ are the estimated variances of the variable $j$ in total observations and in cluster $v$ respectively, $\hat{\pi}_{v j l}$ are the estimated probabilities of distribution of the categorical variables $a_{j}$ in cluster $v, N_{v j l}$ is the number of observations that take $l$ category within cluster $v$ for variable $j$ (Schiopu, 2010).

\section{Results}

We started the study with a correlation analysis between the explanatory variables, in order to avoid multicollinearity. Based on the results we decided to eliminate from the logistic regression model five variables, strongly corelated with others: vaccines are important to protect not only yourself but also others; vaccination of other people is important to protect those that cannot be vaccinated; no information on vaccination in the media in the past six months; information on vaccination in the media in the past six months from newspapers or magazines; and information on vaccination in the media in the past six months from other Internet sites.

The results of the binary logistic regression demonstrated that self-vaccination is determined by citizens' perceptions about vaccination and the sociodemographic profile of the individuals (see Table 1).

One of the most important result of the regression analysis is that the personal decision to be vaccinated is related to the opinion that vaccines are effective, people who believe in the effectiveness of vaccines being 2.2 times more likely to get vaccinated.

Also, the knowledge about vaccination is very important. We used in our analysis a vaccine knowledge index that comprises four aspects: i) vaccines overload and weaken the immune system, ii) vaccines can cause the disease against which they protect; iii) vaccines can often produce serious side effects, and iv) vaccines are rigorously tested before being authorized for use. The index is categorical, with 3 possible values: high knowledge about vaccination, medium, and low knowledge. The logistic regression analysis pointed out that a higher level of vaccine knowledge is associated with a higher likelihood of being vaccinated. 
T able 1

Results of the Logistic Regression Estimation

\begin{tabular}{|c|c|c|c|c|}
\hline \multicolumn{2}{|l|}{ Explanatory variables } & $\begin{array}{c}\text { B } \\
\text { (Coefficient) }\end{array}$ & Wald test & $\begin{array}{c}\operatorname{Exp}(\mathrm{B}) \\
(\text { Odds ratio })\end{array}$ \\
\hline \multicolumn{2}{|l|}{ Vaccine effectiveness } & $0.785^{*}$ & 151.589 & 2.192 \\
\hline Vaccine knowledge index & $\begin{array}{l}\text { High knowledge } \\
\text { Medium knowledge } \\
\text { Low knowledge (ref) }\end{array}$ & $\begin{array}{l}0.743^{*} \\
0.470^{*}\end{array}$ & $\begin{array}{r}143.859 \\
58.555\end{array}$ & $\begin{array}{l}2.103 \\
1.600\end{array}$ \\
\hline \multicolumn{2}{|c|}{ It is important for everybody to have routine vaccinations } & $0.737^{*}$ & 141.813 & 2.091 \\
\hline \multicolumn{2}{|c|}{ Vaccines are only important for children } & $0.120^{*}$ & 14.934 & 1.127 \\
\hline \multicolumn{2}{|c|}{ Not getting vaccinated can lead to serious health issues } & $0.490^{*}$ & 79.640 & 1.632 \\
\hline \multicolumn{2}{|c|}{ Vaccine info sources: family } & 0.014 & 0.076 & 1.014 \\
\hline \multicolumn{2}{|l|}{ Vaccine info sources: friends } & -0.094 & 1.717 & 0.910 \\
\hline \multicolumn{2}{|c|}{$\begin{array}{l}\text { Vaccine info sources: general practitioner, a doctor, } \\
\text { or a pediatrician }\end{array}$} & $0.272 *$ & 47.119 & 1.312 \\
\hline \multicolumn{2}{|c|}{$\begin{array}{l}\text { Vaccine info sources: other healthcare workers } \\
\text { (nurses, specialist doctors) }\end{array}$} & $0.137^{*}$ & 19.180 & 1.147 \\
\hline \multicolumn{2}{|c|}{ Vaccine info sources: pharmacists } & $0.072 * *$ & 3.949 & 1.075 \\
\hline \multicolumn{2}{|c|}{ Vaccine info sources: online social networks } & -0.023 & 0.133 & 0.977 \\
\hline \multicolumn{2}{|c|}{ Vaccine info sources: other Internet sites } & $0.153^{*}$ & 12.064 & 1.166 \\
\hline \multicolumn{2}{|c|}{ Vaccine info sources: the health authorities } & $0.237 *$ & 51.908 & 1.267 \\
\hline \multicolumn{2}{|c|}{$\begin{array}{l}\text { Vaccination programs should be coordinated: at international } \\
\text { level }\end{array}$} & $0.138^{*}$ & 18.364 & 1.148 \\
\hline \multicolumn{2}{|c|}{$\begin{array}{l}\text { Vaccination programs should be coordinated: at European } \\
\text { level }\end{array}$} & 0.047 & 2.205 & 1.048 \\
\hline \multicolumn{2}{|c|}{$\begin{array}{l}\text { Vaccination programs should be coordinated: at national } \\
\text { level }\end{array}$} & -0.024 & 0.580 & 0.977 \\
\hline \multicolumn{2}{|c|}{$\begin{array}{l}\text { Vaccination programs should be coordinated: at regional } \\
\text { or local level }\end{array}$} & -0.029 & 0.560 & 0.972 \\
\hline \multicolumn{2}{|c|}{$\begin{array}{l}\text { There should be no vaccination programs, it is a personal } \\
\text { choice }\end{array}$} & $-0.233^{*}$ & 11.956 & 0.792 \\
\hline \multicolumn{2}{|c|}{$\begin{array}{l}\text { Information on vaccination in the media in the past six months: } \\
\text { Yes, on TV }\end{array}$} & $0.105^{*}$ & 12.141 & 1.111 \\
\hline \multicolumn{2}{|c|}{$\begin{array}{l}\text { Information on vaccination in the media in the past six months: } \\
\text { Yes, on the radio }\end{array}$} & $0.199 *$ & 27.293 & 1.221 \\
\hline \multicolumn{2}{|c|}{$\begin{array}{l}\text { Information on vaccination in the media in the past six months: } \\
\text { Yes, on online social networks }\end{array}$} & $0.135^{*}$ & 8.814 & 1.145 \\
\hline \multicolumn{2}{|c|}{ Improve access to healthcare: more research } & 0.024 & 0.299 & 1.024 \\
\hline \multicolumn{2}{|c|}{ Improve access to healthcare: more affordable treatments } & -0.038 & 0.924 & 0.963 \\
\hline \multicolumn{2}{|c|}{ Improve access to healthcare: more staff in rural area } & $0.128 *$ & 9.573 & 1.137 \\
\hline \multicolumn{2}{|c|}{ Improve access to healthcare: more physicians / doctors } & 0.017 & 0.174 & 1.018 \\
\hline \multicolumn{2}{|c|}{$\begin{array}{l}\text { Improve access to healthcare: more money allocated } \\
\text { to healthcare }\end{array}$} & -0.013 & 0.106 & 0.987 \\
\hline \multicolumn{2}{|c|}{ Improve access to healthcare: more medical establishments } & -0.072 & 2.312 & 0.931 \\
\hline \multicolumn{2}{|c|}{ Gender } & $0.074^{* *} *$ & 6.517 & 1.077 \\
\hline Age & $\begin{array}{l}15-24 \text { years } \\
25-39 \text { years } \\
40-54 \text { years } \\
55 \text { years and older }(\mathrm{r} e f)\end{array}$ & $\begin{array}{r}0.245^{*} \\
-0.210^{*} \\
-0.336^{*}\end{array}$ & $\begin{array}{r}7.720 \\
23.072 \\
68.159\end{array}$ & $\begin{array}{l}1.278 \\
0.810 \\
0.714\end{array}$ \\
\hline Education & $\begin{array}{l}\text { over } 20 \text { years } \\
16-19 \text { years } \\
\text { up to } 15 \text { years }(\text { ref })\end{array}$ & $\begin{array}{l}-0.090^{* * * *} \\
-0.394 *\end{array}$ & $\begin{array}{r}3.575 \\
79.001\end{array}$ & $\begin{array}{l}0.914 \\
0.675\end{array}$ \\
\hline Marital status & & -0.022 & 0.499 & 0.978 \\
\hline Employed & & $-0.126^{*}$ & 12.337 & 0.882 \\
\hline Type of community & $\begin{array}{l}\text { Rural } \\
\text { Small town } \\
\text { Large town }\end{array}$ & $\begin{array}{l}0.029 \\
0.068 * * *\end{array}$ & $\begin{array}{l}0.614 \\
3.607\end{array}$ & $\begin{array}{l}1.029 \\
1.070\end{array}$ \\
\hline Difficulties in paying bills & $\begin{array}{l}\text { Most of the time } \\
\text { From time to time } \\
\text { Almost never/never }\end{array}$ & $\begin{array}{l}-0.321^{*} \\
-0.346^{*}\end{array}$ & $\begin{array}{l}32.593 \\
93.393\end{array}$ & $\begin{array}{l}0.726 \\
0.707\end{array}$ \\
\hline Constant & & $-2.798^{*}$ & 489.514 & 0.061 \\
\hline
\end{tabular}

Note: $* 1 \%$ Significance level, $* * 5 \%$ significance level, $* * * 10 \%$ significance level.

Source: Authors calculations in SPSS, using data from Eurobarometer 91.2. 
A person's opinion on the importance of vaccination proved to be a statistically significant influencing factor for the vaccination decision. The results indicated that people who consider routine vaccination to be important are 2 times more likely to get vaccinated than those who do not share this view. The opinion that not getting vaccinated can lead to serious health problems increases the probability of vaccination by $63.2 \%$. Even those who believe that vaccination is only important for children are $12.7 \%$ more likely to get vaccinated.

Considering the sources of information regarding vaccines, the results of the econometric estimation highlighted that information from medical sources contributes to the vaccination decision. Thus, people who get information from their general practitioner, doctor, or a pediatrician; from other healthcare workers (nurses, specialist doctors), from pharmacists or from the health authorities have a higher probability of getting vaccinated. Vaccine information from online social networks is not a statistically significant factor in a person's decision to get vaccinated. We also included in the analysis variables related to the vaccination program (what vaccines a person should receive and at what time in life), more precisely the level at which this program should be coordinated. People who believe that the vaccination program should be coordinated and harmonized internationally are $14.8 \%$ more likely to get vaccinated. On the contrary, those who consider vaccination to be a personal choice and there should be no vaccination programs are associated with a lower likelihood of being vaccinated.

The media is an important source of information in recent days, so we included in the analysis the influence of information broadcast through the main media channels on the vaccination decision. The results indicated that individuals who find information about vaccination on TV, on the radio or on online social networks are more likely to be vaccinated.

A more general aspect investigated in relation to vaccination was the opinion of individuals regarding the ways to improve access to healthcare (more research, more affordable treatments, more medical staff in rural areas, more doctors, more money allocated to healthcare, more medical establishments), but most of these opinions proved not to be statistically significant in influencing the decision to get vaccinated. Only the variant according to which the access to healthcare would be improved by increasing medical staff in rural areas has a slight influence, indicating that people who share this opinion have higher chances to get vaccinated.

When investigating the personal characteristics that describe the profile of the individual who decides to get vaccinated, we found that gender, age, education, employment status, income, and type of community are important socio-demographic variables that influence the vaccination decision. 
The logistic regression results indicated that men are more likely to be in favor of vaccination. Regarding age, we obtained that young people (15 - 24 years) have higher chances to get vaccinated, compared to people aged 55 and over; in contrast, those from $25-39$ and $40-54$ years groups were less likely to be vaccinated than those aged 55 years and over. Education also has an influence on vaccination, higher levels of education being associated with a lower likelihood of being vaccinated. Marital status is not a significant factor influencing the decision of getting vaccinated. As for the employment status, we found that the employed or self-employed are less likely to get vaccinated compared to people not having a job.

When considering the type of community a person lives in, the results pointed out that people living in small or middle towns are more likely to get vaccinated compared to those living in large towns. For the rural area, the coefficient was not statistically significant.

We wanted to analyze the influence of income on vaccination, therefore we included as explanatory variable a proxy for income: the difficulties faced in paying bills. The econometric results indicated that people facing difficulties in paying bills most of the time or from time to time have lower chances of getting vaccinated compared to those who do not have financial difficulties.

TwoStep Cluster Analysis demonstrated that there are larger differences between groups of people than between European countries concerning vaccination. TwoStep Cluster algorithm resulted in grouping European citizens in 15 clusters which demonstrates the increased heterogeneity in terms of people knowledge about the vaccine, attitudes towards vaccination and confidence in the effectiveness of vaccines (Annex 1).

The quality of the grouping is demonstrated by the Silhouette measure whose value is close to 1 and which can be used in assessing the validity of clustering (Rousseeuw, 1987).

From the 15 clusters, we selected and analyzed in terms of demographic and socio-economic characteristics, two groups with extreme attitudes: favorable to vaccination, with high knowledge and trust in vaccine effectiveness (Cluster 1, comprising 4,654 respondents) and unfavorable to vaccination, with low knowledge and rather skeptical about vaccine effectiveness (Cluster 12, comprising 750 respondents).

Comparing the characteristics of European citizens in cluster 1 (with favorable attitudes towards vaccination) with those included in cluster 12 (with unfavorable attitude), we noticed certain differences in terms of the share of men (over $46 \%$ in cluster 1 , compared to $39 \%$ in cluster 12 ), young people, with ages between 16 and 24 years (almost 10\% in cluster 1, compared to $4 \%$ in cluster 12), 
individuals with high level of education, 20 years of education or older (over $48 \%$ in cluster 1, compared to $30 \%$ in cluster 12) (Figure 1).

Fig u r e 1

Demographic and Social Characteristics of the Respondents from Cluster 1 and 12
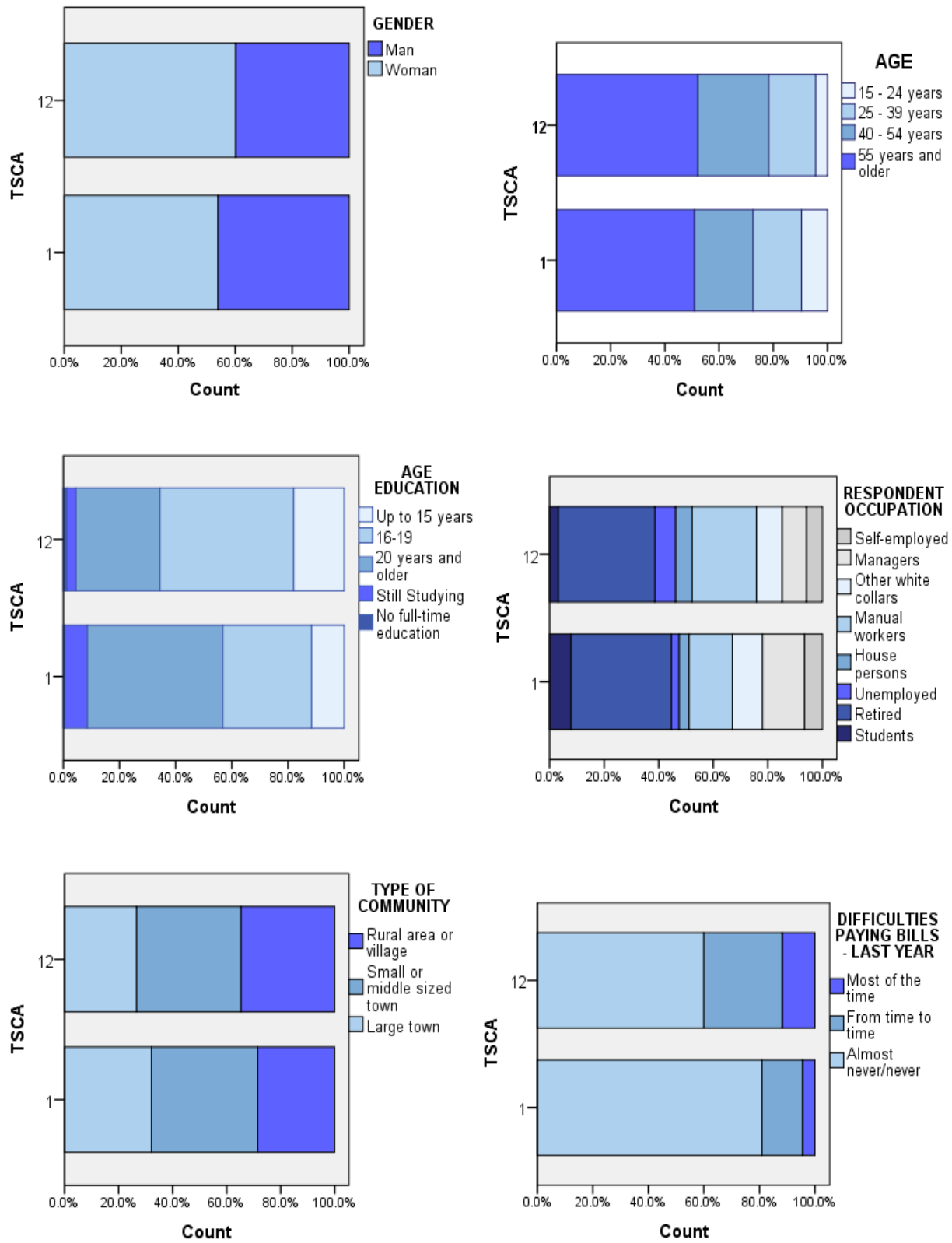

Source: Authors' computation using IBM SPSS Statistics 21. 
In addition, the main features of the citizens in cluster 12, with unfavorable attitudes towards vaccination are: rather women (over 60\% of the total population of cluster 12), over 40 years old (almost $80 \%$ of the respondents in cluster 12), with a medium or low level of education (approximately $66 \%$ of the total European citizens in cluster 12), mostly retirees or manual workers (almost $60 \%$ of the total population in cluster 12), coming from rural areas or small cities (over $70 \%$ of total population in cluster 12) and with a lower level of income (approximately $60 \%$ of cluster 12 population) (Figure 1).

Many of the respondents who grouped in cluster 1 come from countries such as: Sweden, Germany or Netherlands. The respondents less favorable to vaccination are coming from countries such as: Lithuania, Estonia or Latvia. Given that statistics have shown that there are large differences between individuals regarding knowledge, attitudes, and behavior towards vaccination, we expect that such differences to occur between countries as well. To test this hypothesis, we have grouped the European Union countries and United Kingdom in clusters. We have included in the TwoStep Cluster analysis the 3 items mentioned above (vaccine_ effectiveness, self_vaccination, and vaccine_knowledge).

When running the TwoStep Cluster algorithm, the 28 countries from our sample have grouped in two clusters. For this solution, the silhouette measure of cohesion and separation was 0.5 . Cluster 1 is the largest containing 20 countries and Cluster 2 is smaller, comprising 8 countries (Annex 2).

Cluster 1 (Austria, Belgium, Bulgaria, Croatia, Cyprus, Czech Republic, Estonia, France, Greece, Hungary, Ireland, Italy, Latvia, Lithuania, Luxembourg, Malta, Poland, Romania, Slovakia and Slovenia) registered, for each of the three variables assessed, a median score lower than the overall median score (for the entirely sample) (Annex 2). Cluster 2 (Denmark, Finland, Germany, Portugal, Spain, Sweden, Netherlands, and United Kingdom) distinguishes from the others by higher median scores for each of the variables: vaccine_effectiveness, vaccine _knowledge and self_vaccination compared to the overall median (Annex 2).

\section{Discussion and Conclusions}

Vaccination is an important component of health policy in each country of the world, while vaccine hesitancy is considered as one of the ten threats to world health (Rousseeuw, 1987). Therefore, in this research we aimed at investigating the factors that shape the attitudes of individuals towards vaccination, so that decision makers could consider the drivers of these attitudes in the conditions of future vaccinations and find tailored public policies for overcoming the Covid-19 pandemic effects. 
The logistic regression, performed on a large sample of European citizens, allowed us to capture certain patterns of perceptions related to vaccination. As highlighted in the literature, knowledge about vaccines (Lewandowska et al., 2020), confidence in their effectiveness and recognition of their importance (Hadjipanayis et al., 2020) are among the key factors shaping the behavior of the population related to vaccination. Certain authorities in which population trusts are also determinants of behavior (Nowak and Cacciatore, 2016): healthcare workers are perceived as the most reliable sources of specialized information, whereas international institutions are the most relevant for coordinating and harmonizing vaccination programs. The media in general, TV, radio and online social networks in particular, play an important role in shaping attitudes toward vaccination (Yaqub et al., 2014). This fact has drawn our attention to the essential role of the healthcare authorities in promptly transmitting accurate and scientifically proven information and to the risk of increasing vaccine hesitancy through false or poor documented news, which spread very quickly through the internet or other means of communication.

Since from the regression analysis we deduced that the most important factors for self-vaccination are knowledge, confidence in vaccines and trust in healthcare personnel, we deepened the investigation by looking for the two most contrasting groups in terms of attitudes towards vaccination.

As confirmed by other recent studies (Lee and Sibley, 2020), we demonstrated that there is a large heterogeneity in terms of citizen perceptions related to vaccination. TwoCluster Analysis emphasized the existence of no less than 15 clusters in which European citizens should be grouped in terms of knowledge, attitudes, and behaviour towards vaccination.

We identified among the 15 clusters two groups with extreme perceptions: favourable and unfavourable to vaccination. What is interesting to note is that the composition of these two groups is not as different as we would expect. Moreover, the second group, unfavourable to vaccination, is also highly heterogeneous in terms of demographic and socio-economic characteristics.

This result led us to assume that there are at least two profiles in terms of people with unfavourable attitudes towards vaccination. The first, which we named the 'vulnerable group' comprises rather women, aged 40 years and over with a medium or low level of education, coming from small towns or rural areas and with a low level of income. The second category includes educated and wealthy people, rather self-employed or white-collars and living in big cities, which also can be less favourable to vaccination and can be classified as the 'group of skeptics'. We named the first category of population vulnerable in terms of vaccination because most likely poverty and lack of education are the 
main reasons why they do not get vaccinated. The second category is that of skeptics, who although they have knowledge about vaccination and access to multiple sources of information, they do not trust in the effectiveness and safety of vaccines in general, and in those who promote them, in particular.

In line with the recent literature (Dubé et al., 2014), cluster analysis showed that Europeans' perceptions about vaccination are not really country-dependent. In terms of knowledge, attitudes, and behaviour towards vaccination, countries were grouped into two categories: the first cluster comprising the countries with people more hesitant in terms of vaccination, whereas the second contains the countries with people more likely to be vaccinated. However, the silhouette measure of cohesion and separation of 0.5 demonstrated that there are greater differences in citizens' perceptions within a country between different social categories than between countries, a strong reason for a uniform and internationally coordinated vaccination policy.

Our analysis suggests that the success of vaccination in the current context of the crisis caused by Covid-19 depends on specific policies designed to target the two main categories of people less prone to vaccination: vulnerable people and skeptics. Measures like continuous training of health professionals which are the most reliable sources of patient information; developing the necessary infrastructure to connect them with patients, including with the help of new ICT; adapting information campaigns to local specificities could be the pillars of an internationally health strategy.

Our analysis demonstrated the need for internationally coordinated action for increasing the confidence of citizens in vaccines and in authorities, but also the need for measures targeting vulnerable groups.

Thereby, our contribution to the literature is twofold: we demonstrated by means of statistical and econometric techniques, the heterogeneity of the perceptions related to vaccination and the need for tailored smart solutions; we also highlighted the existence of at least two profiles of people less favourable to vaccination: vulnerable and skeptic. Starting from this delimitation, policy makers can find the best solutions to increase vaccination uptake and confidence in vaccines. These solutions are also of at least two types: those targeting vulnerable people, aimed at increasing the knowledge and accessibility of the vaccine; and those targeting the group of skeptics, aimed at increasing confidence in vaccination and in healthcare authorities.

Some limitations of our work need to be considered. We investigated the attitudes of the European citizens towards vaccination as expressed in 2019, before the outbreak of the current Covid-19 crisis. We are aware that the attitudes may have transformed under the effects of the health crisis and the huge 
amount of information. However, in this paper we have captured the attitudes unmodified by recent developments, and in a new research we can compare and evaluate the changes.

\section{References}

AKMATOV, M. K. - RUEBSAMEN, N. - DEYNEKO, I. V. - KARCH, A. - MIKOLAJCZYK, R. T. (2018): Poor Knowledge of Vaccination Recommendations and Negative Attitudes towards Vaccinations Are Independently Associated with Poor Vaccination Uptake among Adults - Findings of a Population-based Panel Study in Lower Saxony, Germany. Vaccine, 36, No. 18 , pp. $2417-2426$.

ANDERSON, R. M. - HEESTERBEEK, H. - KLINKENBERG, D. - HOLLINGSWORTH, T. D. (2020): How will Country-based Mitigation Measures Influence the Course of the COVID-19 Epidemic? Lancet, 395, No. 10228, pp. 931 - 934.

BACHER, J. - WENZIG, K. - VOGLER, M. (2004): SPSS TwoStep Cluster - a First Evaluation. [Online.] [Accessed on 12 August 2020.] Available at: <https://nbn-resolving.org/urn:nbn:de:0168-ssoar-327153>.

BELLI, P. C. - BUSTREO, F. - PREKER, A. (2005): Investing in Children's Health: What Are the Economic Benefits? Bull World Health Organ., 83, No. 10, pp. 777 - 784.

BLOOM, D. E. - CANNING, D. - JAMISON, D. T. (2004): Health, Wealth, and Welfare. Finance Dev., 41 , No. 1 , pp. $10-15$.

COLLETT, D. (2002): Modelling Binary Data. London: Chapman \& Hall/CRC.

de FIGUEIREDO, A. - SIMAS, C. - KARAFILLAKIS, E. - PATERSON, P. - LARSON, H. J. (2020): Mapping Global Trends in Vaccine Confidence and Investigating Barriers to Vaccine Uptake: A Large-scale Retrospective Temporal Modelling Study. Lancet, 396, No. 10255, pp. 898 - 908. Available at: 〈https://doi.org/10.1016/S0140-6736(20)31558-0〉.

DUBÉ, E. - GAGNON, D. - NICKELS, E. - JERAM, S. - SCHUSTER, M. (2014): Mapping Vaccine Hesitancy - Country-specific Characteristics of a Global Phenomenon. Vaccine, 32, No. 49, pp. 6649 - 6654. Available at: 〈https://doi.org/10.1016/j.vaccine.2014.09.039>.

EUROPEAN COMMISSION (2019): Eurobarometer 91.2. [Online.] [Accessed on 12 June 2020.] Available at: <https://www.da-ra.de/dara/study/web_show?res_id=688157\&lang=en\&mdlang= en\&detail=true $>$.

GHERGHINA, I. - COCHINO, A. V. - ALOMAN, M. M. - COVĂCESCU, M. S. - CONSTANTIN, A. T. (2019): Vaccination Management of Children in Romania. Romanian Journal of Pedriatics, LXVIII, Supplement.

GIANG, T. L. - VO, D. T. - VUONG, Q. H. (2020): COVID-19: A Relook at Healthcare Systems and Aged Populations. Sustainability, 12, No. 4200, pp. 1 - 10. [Online.] [Accessed on 11 September 2020.] Available at: 〈https://doi.org/10.3390/su12104200>.

GILlET, A. - BROSTAUX, Y. - PALM, R. (2010): Principaux modèles utilisés en régression logistique. Biotechnol. Agron. Soc. Environ., 15, No. 3, pp. 425 - 433.

HADJIPANAYIS, A. et al. (2020): Vaccine Confidence among Parents: Large Scale Study in Eighteen European Countries. Vaccine, 38, No. 6, pp. 1505 - 1512.

IBM (2020): TwoStep Cluster. [Online.] [Accessed on 12 August 2020.] Available at: <https://www.ibm.com/support/knowledgecenter/en/SSLVMB_23.0.0/spss/base/idh_twostep_ main.html>.

La TORRE, G. - SCALINGI, S. - GARRUTO, V. - SICLARI, M. - CHIARINI, M. - MANNOCCI, A. (2017): Knowledge, Attitude and Behaviours towards Recommended Vaccinations among Healthcare Workers. Healthcare, 5, No. 1, pp. 1 - 17, Article No. UNSP 13. [Online.] [Accessed on 12 September 2020.] Available at: <https://doi.org/10.3390/healthcare5010013>. 
LA, V. P. et al. (2020): Policy Response, Social Media and Science Journalism for the Sustainability of the Public Health System Amid the COVID-19 Outbreak: The Vietnam Lessons. Sustainability, 12, No. 7, pp. 1 - 27, Article No. 2931. [Online.] [Accessed on 12 September 2020.] Available at: <https://doi.org/10.3390/su12072931>.

LACKO, R. - MARKOVIČ, P. - ŠAGÁTOVÁ, S. - HAJDUOVÁ, Z. (2020): Evaluation and Development of Environmental and Health Efficiency: Case of V4 Countries. Ekonomický časopis/Journal of Economics, 68, No. 10, pp. 1040 - 1056. Available at: <https://doi.org/10.31577/ekoncas.2020.10.04>.

LEE, C. H. J. - SIBLEY, C. G. (2020): Attitudes toward Vaccinations Are Becoming More Polarized in New Zealand: Findings from a Longitudinal Survey. EClin. Med., 6, No. 23, pp. 1 - 9, Article No. 100387. [Online.] DOI: 10.1016/j.eclinm.2020.10038.

LEWANDOWSKA, A. - LEWANDOWSKI, T. - RUDZKI, G. - RUDZKI, S. - LASKOWSKA, B. (2020): Opinions and Knowledge of Parents Regarding Preventive Vaccinations of Children and Causes of Reluctance toward Preventive Vaccinations. Int J Environ Res Public Health, 17, No. 10, pp. 1 - 13, Article No. 3694. [Online.] [Accesed on 10 June 2020.] Available at: <https://www.mdpi.com/1660-4601/17/10/3694>.

MARINESCU, C. (2020): Corona Economy - from Crisis to Restoring Confidence. [Online.] [Accessed on 12 October 2020.] Available at: $<$ http://cosmin-marinescu.ro/corona-economy-de-la-criza-la-refacerea-increderii/>.

NATIONAL ACADEMIES of SCIENCES (2020): Engineering, and Medicine. Discussion Draft of the Preliminary Framework for Equitable Allocation of COVID-19 Vaccine. [Accessed on 28 September 2020.] Available at: <https://www.aha.org/lettercomment/2020-09-08-aha-comments-nasems-discussion-draftpreliminary-framework-equitable>.

NITSCH-OSUCH, A. - JAGIELSKA, A. - BRYDAK, L. B. (2020): Influenza Vaccination Coverage Rates in the General Population and Risk Groups: A Review of the Current International Situation. Postepy Higieny I Medycyny Doswiadczalnej, 72, No. 1138 - 1147, pp. 1138 - 1147.

NOWAK, G. J. - CACCIATORE, M. A. (2016): Parents' Confidence in Recommended Childhood Vaccinations: Extending the Assessment, Expanding the Context. Human Vaccines \& Immunotherapeutics, 13, No. 3, pp. $687-700$.

PRIMC, K. - SLABE-ERKER, R. (2020): The Success of Public Health Measures in Europe during the COVID-19 Pandemic. Sustainability, 12, No. 10, pp. 1 - 13, Art. No. 4321. [Online.] [Accessed on 11 August 2020.] Available at: <https://doi.org/10.3390/su12104321>.

RAPPUOLI, R. (2014): A New Horizon for the Vaccine. [Online.] [Accessed on 12 August 2020.] Available at: 〈https://ec.europa.eu/research/health/pdf/event17/s2-2-rino-rappuoli_en.pdf〉.

ROUSSEEUW, P. J. (1987): Silhouettes: A Graphical Aid to the Interpretation and Validation of Cluster Analysis. Journal of Computational and Applied Mathematics, 20, November, pp. 53 - 65.

SALMON, D. - DUDLEY, M. Z. (2020): It Is Time to Get Serious about Vaccine Confidence. The Lancet, 396, No. 10255 , pp. $870-871$.

SCHIOPU, D. (2010): Applying Two Step Cluster Analysis for Identifying Bank Customer's Profile. Economic Insights - Trends and Challenges, 62, No. 3, pp. 66-75.

WEERAMANTHRI, T. S. - BAILIE, R. S. (2015): Grand Challenges in Public Health Policy. Frontiers in Public Health, 3, No. 29, pp. 1 - 4. [Accessed on 12 May 2020.] Available at: <https://doi.org/10.3389/fpubh.2015.00029>.

WORLD HEALTH ORGANIZATION (WHO) Immunization Rates, Fact Sheet 2018. [Online.] [Accessed on 12 August 2020.] Available at: $<$ http://www.who.int/mediacentre/factsheets/fs378/en/>.

YAQUB, O. - CASTLE-CLARKE, S. - SEVDALIS, N. - CHATAWAY, J. (2014): Attitudes to Vaccination: A Critical Review. Social Science \& Medicine, 112, July, pp. 1 - 11. Available at: 〈https://doi.org/10.1016/j.socscimed.2014.04.018>. 


\section{A n n e x e s}

A n n e x 1

Cluster Analysis Results

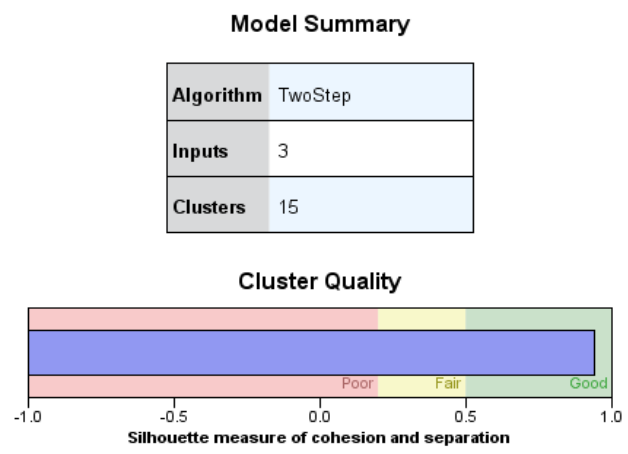

Cluster Comparison

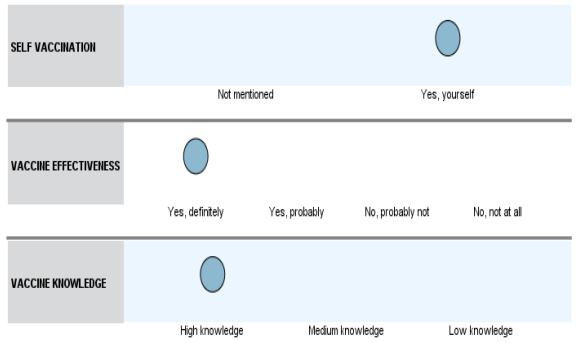

Cluster Comparison

II 12

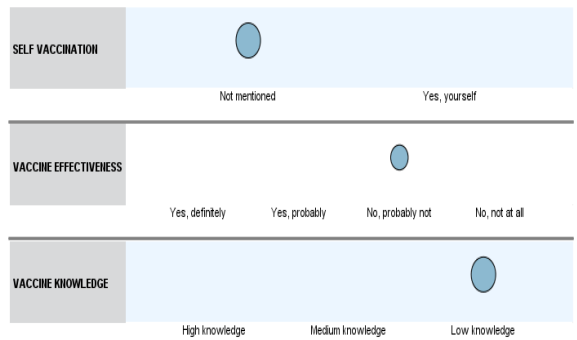

Source: Authors' computation using IBM SPSS Statistics 21. 
A n n ex 2

Clusters' Comparison in Terms of Vaccination Attitude
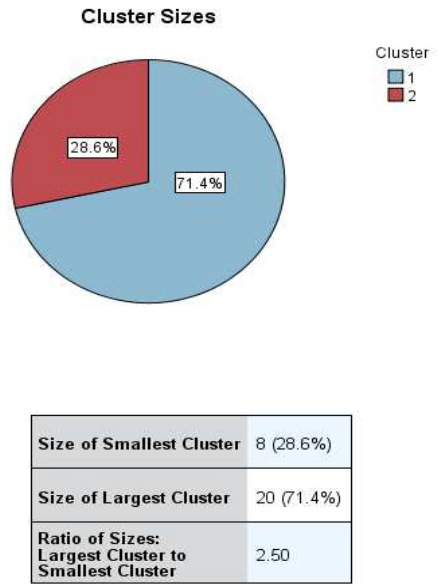

Cluster Comparison

1

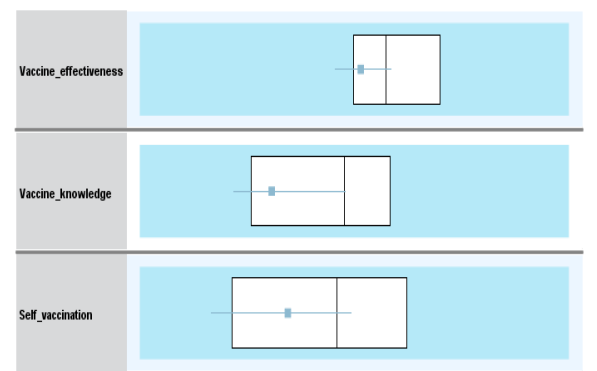

Cluster Comparison

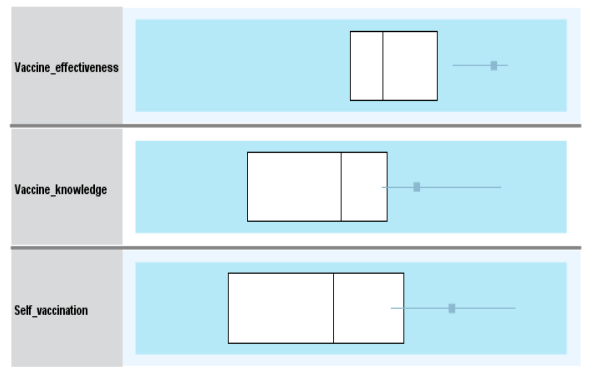

Source: Authors' computation using IBM SPSS Statistics 21. 